\title{
Consumption of Nuts in the Prevention of Cardiovascular Disease
}

\author{
Joan Sabaté • Michelle Wien
}

Published online: 3 October 2013

(C) Springer Science+Business Media New York 2013

\begin{abstract}
The leading cause of morbidity and mortality in highly developed and low- and middle-income nations is cardiovascular disease (CVD). The establishment of healthy dietary patterns is one of the cornerstones of CVD prevention, and nuts have emerged as favorable components of dietary patterns associated with reducing the CVD epidemic. The etiological connection between nuts for the prevention of CVD is based upon several lines of evidence. First, nuts are nutrient-dense whole foods that contain a distinctive mix of macronutrients, specific micronutrients and non-nutrients that have been associated with cardioprotection. Second, numerous human feeding trials have demonstrated that nut intake improves the serum lipid profile, reduces oxidation and inflammation, and improves vascular reactivity. Third, nut consumption is consistently associated with a reduced risk of CVD in many epidemiological studies. Lastly, a recent large randomized clinical trial conducted in Spain demonstrated that consuming mixed nuts daily lowers CVD risk by $30 \%$.
\end{abstract}

Keywords Nuts · Lipids · Cholesterol · Fatty acids · Phytochemicals · Antioxidants - Cardiovascular · Mortality · Hypertension · Inflammation · Endothelial function

J. Sabaté $(\bowtie) \cdot$ M. Wien

Department of Nutrition, School of Public Health, Loma Linda

University, 24951 N. Circle Drive, Nichol Hall 1102,

Loma Linda, CA 92350, USA

e-mail: jsabate@1lu.edu

M. Wien

e-mail: mwien@1lu.edu

J. Sabaté

Department of Epidemiology and Biostatistics, Loma Linda

University, Loma Linda, CA, USA

\section{Introduction}

Significant strides have been made since the 1970s to reduce the complications and mortality associated with cardiovascular disease (CVD) in highly developed nations. However, CVD still remains the leading cause of deaths worldwide due to the concurrent rise and disproportionate burden of CVD in low- and middle-income nations [1•]. Advancing age, gender and family history are indisputable risk factors that contribute to the development of CVD; therefore, modifiable lifestyle risk factors are targeted in public health messages for the primary prevention of CVD. The establishment of healthy dietary patterns is one of the cornerstones of CVD prevention, and tree nuts have emerged as favorable components of healthy dietary patterns associated with diminishing CVD risk.

In the nut family, 10 are botanically classified as tree nuts: almonds, Brazil nuts, cashews, chestnuts, hazel nuts, macadamias, pecans, pine nuts, pistachios, and walnuts. Chestnuts differ significantly from the other nine common tree nuts due to their higher carbohydrate content, whereas peanuts, which are botanically classified as legumes, have a very similar nutrient profile to the nine common tree nuts. Thus, for this review paper we are operationally defining "nuts" to include the nine common tree nuts plus peanuts.

\section{Composition of Nuts and CVD}

In light of their unique macronutrient, micronutrient and nonnutrient bioactive constituents [2], nuts are nutrient-dense whole foods that confer cardioprotection. Nuts contain significantly larger amounts of unsaturated fatty acids (UFA), e.g., monounsaturated fatty acids (MUFA) and polyunsaturated fatty acids (PUFA), as compared to saturated fatty acids (SFA), which results in a preferential UFA:SFA ratio of 
$\sim 3: 12$ (Table 1). Further, walnuts contain the highest level of alpha-linolenic acid (ALA) among all plant sources, which is the n-3 fatty acid that has been shown to exert cardioprotection through the reduction of platelet aggregation, vasoconstriction and inflammation [3, 4]. Nuts are also a good source of plant protein $(\sim 8-26 \mathrm{~g}$ per $100 \mathrm{~g})$ and most nuts are rich sources of the amino acid L-arginine [5], which is the precursor of the powerful endogenous vasodilator nitric oxide (NO) [6]. In light of their high arginine content, a preferentially low arginine to lysine ratio between 0.01 and 0.57 is found in nuts, which can contribute towards reducing plasma low density lipoprotein (LDL) levels $[7,8]$. The very low glycemic index of nuts $(<15)$ is due in part to their low carbohydrate content (12-30 g per $100 \mathrm{~g})$ in the context of their dietary fiber content $(\sim 3-12 \mathrm{~g}$ per $100 \mathrm{~g})$ [9].

Nuts contain significant amounts of bioavailable antioxidants, i.e., tocopherols and bioactive compounds, including phytochemicals, phytoestrogens and phytosterols, that have the ability to be cardioprotective through various mechanisms [10]. Notably, almonds and hazelnuts have the highest levels of $\alpha$-tocopherol, whereas pecans, pistachios and walnuts contain significant levels of $\gamma$-tocopherol. Additionally, the phytosterol content of nuts may partially inhibit the absorption of dietary cholesterol and recirculating endogenous biliary cholesterol in the intestinal lumen, and may be associated with reductions in plasma cholesterol and CVD prevention [11].

Nuts are rich in several cardioprotective minerals. Reductions in blood pressure were observed from the intake of the Dietary Approaches to Stop Hypertension (DASH) dietary pattern [12], which included nuts and other nutrient-dense whole foods. More specifically, a low sodium intake in the context of a high intake of magnesium, potassium and calcium using the DASH pattern was associated with reductions in systolic and diastolic blood pressure by 6 and $3 \mathrm{~mm} \mathrm{Hg}$, respectively, in patients with pre-hypertension, and reductions of 11 and $5 \mathrm{~mm} \mathrm{Hg}$, respectively, in hypertensive patients. Further, unprocessed nuts are whole foods that contain negligible amounts of sodium, and many are rich sources of magnesium, potassium and copper [13], which are associated with the prevention of ventricular arrythmias and reductions in arterial hypertension $[14,15]$.

\section{Summary Statement for Composition of Nuts and CVD}

The composition of nuts shown in Table 1 lists several of the macronutrients, specific micronutrients and non-nutrients that have been associated with cardioprotection. To achieve optimal health outcomes (e.g., CVD reduction), one of the recommendations given in the 2010 Report of the Dietary Guidelines for Americans is to shift to a more plant-based dietary pattern that includes nuts. Thus, unprocessed nuts are an appropriate whole food to include within a prudent diet to decrease the prevalence of CVD in populations.

\section{Nut Consumption Intervention Trials and CVD Risk Factor Outcomes}

Over 40 nut feeding trials in humans have been conducted worldwide since the findings from a tightly controlled metabolic feeding study performed at Loma Linda University were published in the New England Journal of Medicine [16]. This investigative team found 12 and $18 \%$ reductions in serum total cholesterol (TC) and LDL cholesterol levels, respectively, from the consumption of a walnut-enriched diet (20\% of energy from walnuts) compared with a healthy American Heart Association "Step One" diet (10\% energy from SFA, $10 \%$ energy from MUFA, $10 \%$ energy from PUFA, and $250 \mathrm{mg}$ cholesterol per day). Ten years later, in response to the emerging scientific

Table 1 Nutrient composition of nine commonly consumed tree nuts and peanuts per $100 \mathrm{~g}$

\begin{tabular}{|c|c|c|c|c|c|c|c|c|c|c|c|c|c|}
\hline & $\mathrm{CHO}, \mathrm{g}$ & Fiber, $\mathrm{g}$ & Protein, $g$ & Fat, $\mathrm{g}$ & SFA, $g$ & MUFA, $g$ & PUFA, $g$ & UFA: SFA & $\alpha$-toc, mg & $\gamma$-toc, mg & $\mathrm{K}, \mathrm{mg}$ & $\mathrm{Mg}, \mathrm{mg}$ & Phytosterol, mg \\
\hline Almonds & 21.67 & 12.2 & 21.22 & 49.42 & 3.731 & 30.889 & 12.070 & 11.51 & 26.22 & 0.65 & 705 & 268 & 31.0 \\
\hline Brazil nuts & 12.27 & 7.5 & 14.32 & 66.43 & 15.137 & 24.548 & 20.577 & 2.98 & 5.73 & 7.87 & 659 & 376 & 21.5 \\
\hline Cashews & 30.19 & 3.3 & 18.22 & 43.82 & 7.783 & 23.797 & 7.845 & 4.07 & 0.90 & 0.03 & 660 & 292 & - \\
\hline Hazelnuts & 16.70 & 9.7 & 14.95 & 60.75 & 4.464 & 45.652 & 7.920 & 12.00 & 15.03 & 0.00 & 680 & 163 & 12.0 \\
\hline Macadamia & 13.82 & 8.6 & 7.91 & 75.77 & 12.061 & 58.877 & 1.502 & 5.01 & 0.54 & 0.00 & 368 & 130 & - \\
\hline Pecans & 13.86 & 9.6 & 9.17 & 71.97 & 6.180 & 40.801 & 21.614 & 10.10 & 1.40 & 24.44 & 410 & 121 & 32.8 \\
\hline Pine nuts & 13.08 & 3.7 & 13.69 & 68.37 & 4.899 & 18.764 & 34.071 & 10.78 & 9.33 & 11.15 & 597 & 251 & 84.1 \\
\hline Pistachios & 27.51 & 10.3 & 20.27 & 45.39 & 5.556 & 23.820 & 13.744 & 6.76 & 2.30 & 22.60 & 1025 & 121 & - \\
\hline Walnuts & 13.71 & 6.7 & 15.23 & 65.21 & 6.126 & 8.933 & 47.174 & 9.16 & 0.70 & 20.83 & 441 & 158 & 18.2 \\
\hline Peanuts & 16.13 & 8.5 & 25.80 & 49.24 & 6.834 & 24.429 & 15.559 & 5.85 & 8.33 & - & 705 & 168 & - \\
\hline
\end{tabular}

Values taken from U.S. Department of Agriculture, Agricultural Research Service. 2012. USDA National Nutrient Database for Standard Reference, Release 25. Nutrient Data Laboratory Home Page, http://www.ars.usda.gov/ba/bhnrc/ndl (accessed 12 July 2013)

CHO carbohydrate, MUFA monounsaturated fatty acid, PUFA polyunsaturated fatty acid, UFA unsaturated fatty acid, SFA saturated fatty acid, $\alpha$-toc alpha-tocopherol, $\gamma$-toc gamma-tocopherol $K$ potassium, $M g$ magnesium 
research that demonstrated the beneficial effects of nut consumption on CVD risk factors, the United States (US) Food and Drug Administration (FDA) released a qualified health claim [17] stating that "Scientific evidence suggests but does not prove that eating 1.5 ounces per day of most nuts, as part of a diet low in saturated fat and cholesterol, may reduce the risk of heart disease."

Human feeding studies have typically been of short-term duration (3-8 weeks) and have been performed on healthy subjects or on moderately hypercholesterolemic patients. The "control" diet for testing the effect of the nut intervention(s) has been the Western (high in saturated fat), Mediterranean, or low saturated fat diet. Also, the most commonly studied nuts have been almonds and walnuts, and there have been no studies conducted using pine nuts to date.

\section{Effect of Nuts on Blood Lipid Levels}

Recently, a pooled analysis of 25 nut feeding trials that were published up to December 2004 was conducted to evaluate the effects of nuts on blood lipid levels, and to determine if these effects remained consistent after stratification by gender, age, body mass index, type of nut, degree of dietary compliance, type of control diet and source of study funding [18 • ]. This pooled analysis of 583 unique subjects and 1,284 observations from seven countries found that the mean intake of $67 \mathrm{~g}$ per day of nuts produced a 5.1, 7.4 and $8.3 \%$ mean estimated reduction in TC, LDL cholesterol and ratio of LDL:HDL, respectively. Null effects were shown for high-density lipoprotein (HDL) cholesterol and triglycerides (TG), with the exception of subjects with TG levels greater than $150 \mathrm{mg} / \mathrm{dl}$ who experienced a significant TG reduction of $20.6 \mathrm{mg} / \mathrm{dl}$ $(10.2 \%)$. An important finding was that the lipid lowering effects of consuming $10,12.2(43 \mathrm{~g} / \mathrm{d}$ as recommended by the USFDA qualified health claim) and $20 \%$ energy from nuts were dose-related. Further, the effects were found to be greater in subjects with a lower baseline BMI $\left(<25 \mathrm{~kg} / \mathrm{m}^{2}\right)$ and lower baseline LDL cholesterol ( $<130 \mathrm{mg} / \mathrm{dl}$ ) level (Fig. 1).

A prior meta-analysis evaluated 13 clinical walnut intervention trials that had been conducted with study durations ranging from four to 24 weeks [19], using a range of 5 to $25 \%$
Fig. 1 Total cholesterol (TC), low-density lipoprotein cholesterol (LDL-C), and LDL-C to high-density lipoprotein cholesterol (HDL-C) ratio response to nut intake by baseline LDL-C level and body mass index (BMI). Data presented from a pooled analysis study of 25 nut intervention trials, for which the mean serving size was $67 \mathrm{~g}$ (2.4 oz) of nuts per day. (Data adapted from Sabaté, et al. JAMA 2010)
Baseline LDL-C Concentration $\mathrm{mg} / \mathrm{dL}$
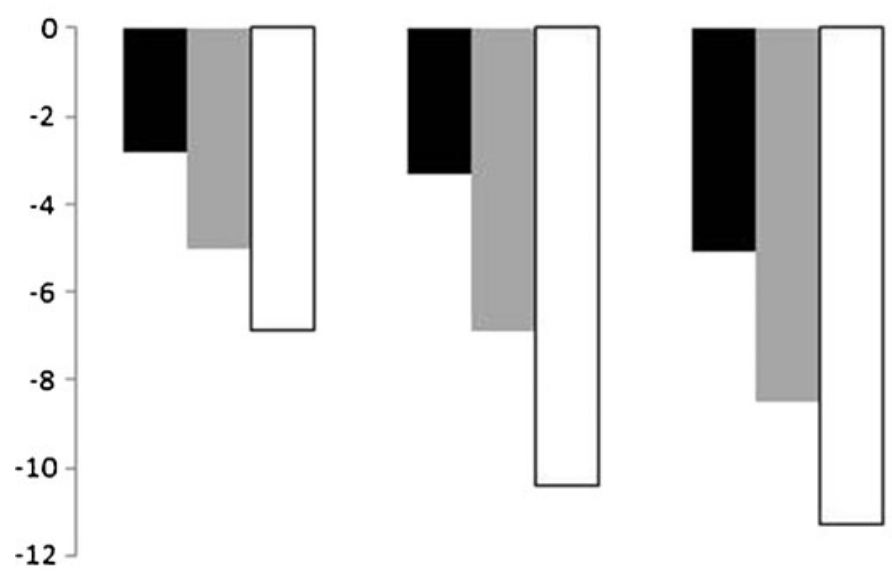

$<130$

$130-160$

$>160$

\section{Baseline BMI $\left(\mathrm{kg} / \mathrm{m}^{2}\right)$}

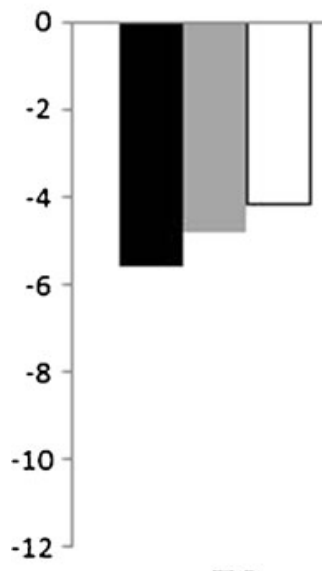

TC

LDL-C

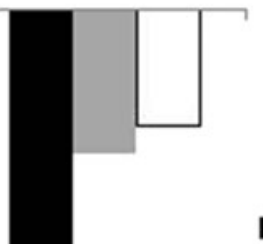

$<25$ 
of daily total energy from walnuts. The findings were in concordance with those of the aforementioned pooled analysis, as the walnut-enriched diets were associated with a $6.7 \%$ decrease in LDL cholesterol level as compared to the control diets, and null results were found for HDL cholesterol and TG.

In light of the increased prevalence of obesity among children and adolescents worldwide [20], research is gradually emerging regarding the effect of nuts on the blood lipid profile and microvascular function of obese children and adolescents. A 16-week, randomized, parallel design trial was recently conducted with 17 obese female adolescents to evaluate the effects of supplementing the diet with Brazil nuts [21••]. Subjects in the intervention group consumed 15-25 g per day of Brazil nuts to achieve $10 \%$ of their daily energy requirements from MUFAs. Compared to the placebo group, Brazil nut intake significantly reduced TC, LDL cholesterol and TG levels by 10.5, 16.1 and $17.7 \%$, respectively. In light of the stable BMI and waist circumference noted during the trial, the investigative team concluded that Brazil nuts are a nutrient-rich whole food that can be used as a supplement to the diet for cardioprotection among obese female adolescents.

\section{Summary Statement for the Effect of Nuts on Blood Lipid Levels}

There is consistently strong evidence from both pooled analysis and meta-analysis showing the positive benefits of nut consumption in a dose-dependent manner for the management of blood lipid levels, with greater benefits being achieved among persons with a BMI less than $25 \mathrm{~kg} / \mathrm{m}^{2}$ and for those with higher LDL cholesterol (>160 mg/dl) levels. Additional studies are warranted to explore the potential synergistic effect of consuming mixed nuts on blood lipid levels in healthy and hypercholesterolemic individuals.

\section{Effect of Nuts on CVD Risk Factors Beyond the Blood Lipid Profile}

As discussed above, nuts are a unique type of plant food that contains a distinctive mix of nutrients and non-nutrient bioactive constituents. Thus, additional cardioprotective benefits from nut consumption beyond improving the blood lipid profile have been extensively studied and summarized by others, specifically on biomarkers of oxidative stress, inflammation and vascular reactivity [22-25].

\section{Biomarkers of Oxidative Stress}

The majority of nuts contain a moderate to high level of MUFA that is not conducive to oxidation. However, nuts contain PUFA that can act as a substrate for oxidation reactions [26]. Walnuts are notably high in PUFA but they inherently contain antioxidants that can potentially mitigate oxidative reactions in biological membranes. It is worth noting that, to date, no human study using walnuts has found any deleterious effects on oxidative status as compared to healthy diets [25].

Findings from early in vivo studies using nut extracts showed reductions of lipid peroxidation or oxidative DNA damage, which were subsequently followed by short- and long-term experimental animal studies that found beneficial effects on antioxidant enzyme activity and lipid oxidation [10, 23]. Randomized controlled feeding trials of 3-8 weeks in duration that have been conducted in humans using MUFArich nuts (e.g., almonds) have found either a reduction in biomarkers of oxidation or null findings [23]. Further, postprandial feeding studies using almonds and walnuts have found either beneficial effects or null findings on postprandial oxidative stress [27-30]. More recent evaluations of the postprandial effect of pecan consumption [31•] and a 4-week feeding trial with pistachios [32•] have both shown reductions in LDL oxidation, the former of which confirmed the bioavailability and contribution of bioactive compounds that can confer beneficial antioxidant defense outcomes.

The recent Prevencion con Dieta Mediterranea (PREDIMED) trial randomized 7,477 subjects (age range 55-80 years) who were at high risk for CVD to (1) an energy-unrestricted Mediterranean diet plus extra-virgin olive oil, (2) an energy-unrestricted Mediterranean diet plus $30 \mathrm{~g}$ per day mixed nuts ( $50 \%$ walnuts, $25 \%$ almonds and $25 \%$ hazelnuts), or (3) an energy-unrestricted control, low-fat diet, and evaluated the groups' oxidized LDL levels after 12 weeks [33]. The PREDIMED investigative team observed that the mixed nut intervention resulted in reduced oxidized LDL levels as compared to the control diet. In contrast to these findings, a parallel design trial using the same mixed nut approach versus a nut-free diet for 12 weeks in 50 adult patients with metabolic syndrome showed null findings for oxidized LDL and other biomarkers, with the exception of reduced DNA damage in the participants consuming the mixed nut diet [34].

\section{Biomarkers of Inflammation}

Null findings have resulted in controlled nut feeding trials in humans that have explored the effects of almond, walnuts and mixed nuts on plasma C-reactive protein (CRP); these results have been previously reviewed by others up to 2008 [25] However, feeding trials using mixed nuts and walnuts alone have found decreased levels of other inflammatory mediators, i.e., intercellular adhesion molecule 1 (ICAM-1), vascular cell adhesion molecule (VCAM)-1, and interleukin (IL)-6 [35, 36]. Additionally, we recently compared two doses of almonds in a tightly controlled feeding trial to a healthy control diet and showed that the two almond diets reduced the levels of CRP and E-selectin in the context of null findings for IL-6 [37॰]. 
In light of the walnut's rich content of the anti-inflammatory fatty acid ALA and the potent phenolic compound ellagic acid, postprandial walnut feeding studies have been conducted in human subjects to evaluate the acute effects of walnut-rich meals and of consuming a walnut-rich, mixed nut diet (over a 3-month period) on the expression of pro-inflammatory molecules in circulating mononuclear cells and/or circulating biomarkers of postprandial inflammation [28, 38, 39]. Walnuts were shown to confer similar or superior postprandial blunting of circulating inflammatory biomarkers, as compared to meals enriched with olive oil $[28,38]$. Additionally, walnut-rich meals reduced the postprandial mRNA expression of IL-6 in circulating blood mononuclear cells [38], and consumption of walnut-rich, mixed nuts diets for 3 months reduced monocyte expression of pro-inflammatory ligands [39].

\section{Endothelial Function}

Several nutrients exist in all nuts that have the potential to influence vascular reactivity, i.e., tocopherols [40], polyphenols [41] and L-arginine [42], which can counteract the transient endothelial dysfunction that occurs when an SFA-rich meal [43] or diet [44-46] is consumed. However, ALA is only found in walnuts and pecans. More specifically, walnuts contain high levels of ALA ( $2.5 \mathrm{~g}$ per $100 \mathrm{~g}$ ) and pecans contain $0.5 \mathrm{~g}$ per $100 \mathrm{~g}$ [45].

Three randomized crossover trials have been conducted to evaluate the effect of postprandial and chronic walnut consumption on endothelial function using flow-mediated dilatation (FMD) in the brachial artery [47]; two trials involved subjects with hypercholesterolemia $[28,36]$ and one trial involved subjects with type 2 diabetes [48••]. Walnuts had beneficial effects on FMD in all three of these trials, which may be due to their high ALA content in the context of their inherently high antioxidant and L-arginine content. Further research using other types of nuts is warranted to determine if their bioactive compounds improve FMD in subjects at high risk of developing CVD.

In light of the consistency of findings showing a beneficial effect from walnut consumption on endothelial function, a specific health claim for walnuts was published in the Official Journal of the European Union in May 2012 [49••]. The walnut-specific health claim states that "Walnuts contribute to the improvement of the elasticity of the blood vessels" and may be used only for food that provides a daily intake of 30 grams of walnuts.

\section{Hypertension and Atrial Fibrillation}

The Shanghai Women's Health Study [50] and the Physicians' Health Study [51•] have both failed to find an association between hypertension and nut consumption, and null findings have also been observed in the small FMD walnut studies that have been previously described. However, the aforementioned PREDIMED trial is the first adequately powered study that has demonstrated reductions in systolic and diastolic blood pressure after consumption of a Mediterranean diet supplemented with mixed nuts versus a low-fat control diet or a Mediterranean diet supplemented with extra-virgin olive oil [35]. These investigators found that the erythrocyte membranes from the subjects consuming mixed nuts daily had reduced cholesterol to phospholipid ratios, and suggested that this could lead to increased membrane fluidity and antihypertensive effects [52]. Additional studies are needed to further elucidate the antihypertensive mechanism(s) related to nut consumption.

Atrial fibrillation is prevalent in the US and European Union [53, 54], and affects approximately 2.3 million and 4.5 million individuals, respectively. Recent findings from the Physicians' Health Study have failed to show an association between incident atrial fibrillation and nut consumption [55•]. Additional prospective studies are warranted, as this cohort solely consisted of male physicians who may have been consuming higher amounts of nuts due to their known health benefits.

\section{Summary Statement for the Effect of Nuts on CVD Risk Factors Beyond the Blood Lipid Profile}

Strong and relatively consistent evidence from ongoing human studies suggests that MUFA-rich nuts may improve oxidative status, and walnuts (PUFA-rich) have either a slightly beneficial or neutral effect. There is consistent evidence from both acute- and long-term nut trials showing reductions in several inflammatory biomarkers. However, inflammatory biomarkers have been secondary outcomes in clinical trials using nut-enriched diets, with the exception of three postprandial walnut feeding trials. These walnut trials are helping to elucidate the anti-inflammatory effects of nuts at the molecular level; however, additional studies are necessary to further evaluate the mechanisms and constituents in nuts that are contributing to these beneficial outcomes. Limited evidence suggests beneficial effects for nut intake on blood pressure, and evidence is currently lacking for an association between nut consumption and incident atrial fibrillation.

\section{Epidemiologic Studies of Nut Consumption, CHD Risk and Cardiovascular Mortality}

In 1992, the Loma Linda University Adventist Health Study-1 (AHS-I) was the first prospective study to report a beneficial effect between a higher frequency of nut intake and reduced CHD incidence in a large cohort of healthy adults (Table 2) 
Table 2 Epidemiological studies on the frequency of nut intake and relative risk of non-fatal myocardial infarction (MI), coronary heart disease (CHD) death and sudden cardiac death

\begin{tabular}{lllll}
\hline Reference & Males/Females & $\begin{array}{l}\text { Number of years } \\
\text { of follow-up }\end{array}$ & Outcomes & Relative Risk \\
\hline Adventist Health Study-1 & $\begin{array}{l}\text { 10,003 Males } \\
\text { 16,740 Females }\end{array}$ & 6 & Non-fatal MI & 0.52 \\
Iowa Women's Health Study & 19,411 Females & 7 & CHD death & 0.62 \\
Nurses' Health Study & 86,016 Females & 14 & CHD death & 0.60 \\
Physicians' Health Study & 21,434 Males & 17 & Non-fatal MI & 0.68 \\
& & & CHD death & 0.61 \\
Combined & 153,604 & 44 & Sudden cardiac death & 0.53 \\
& & & CHD death & 0.77 \\
\hline
\end{tabular}

[56]. The Nurses' Health Study [57], Physicians' Health Study [58], and the Iowa Women's Health Study [59] subsequently reported similar effects from frequent nut consumption in their large US cohorts, which suggests that frequent nut consumption in the context of a US dietary pattern confers cardioprotection.

A summary of the combined evidence from the four USbased cohort studies was reported for the relative risk (RR) of fatal coronary heart disease (CHD) using the median of each nut frequency consumption category, which showed a $37 \%$ decreased RR for those consuming nuts in the highest frequency intake group (more than four times per week) compared to the lowest nut intake group (never or seldom consume nuts) [60]. Further, a clear dose-response gradient was found in each of the four studies, with an $8.3 \%$ average reduction in RR of CHD death for each weekly serving of nuts. Using multivariate analysis, a more recent pooled analysis of these four studies found a similar RR of 0.65 (95\% CI, 0.47-0.89) between the highest nut intake group and total $\mathrm{CHD}$, and an RR of 0.61 (95\% CI, 0.35-1.05) for fatal CHD among participants consuming nuts five or more times per week [61]. In all four studies, the effect of nut consumption frequency and total CHD or fatal CHD was independent of gender, age, BMI, alcohol intake, smoking, presence of CVD risk factors and nutrient characteristics.

Although the primary aim of the PREDIMED trial was to examine the efficacy of two Mediterranean diets as compared to a low-fat control diet on primary CVD prevention (see next section below), the PREDIMED investigative team conducted an additional longitudinal cohort study on 7,216 of the participants using baseline consumption of six specific types of nuts commonly consumed in Spain. Consumption of these nuts (almonds, peanuts, hazelnuts, pistachios and pine nuts; and, walnuts as a separate food item), treated as the exposure, was evaluated for the following three outcomes: total mortality; cardiovascular mortality only; and, cancer mortality only [62••]. Compared to participants that never consumed nuts, those participants that consumed $>3$ weekly servings of total nuts, walnuts or other nuts (excluding walnuts) had significant reductions in cardiovascular mortality of 55, 47 and $58 \%$, respectively. These recent findings are similar to the aforementioned prospective studies that reported significant risk reductions ranging between 30 and $57 \%$ between frequent nut consumption and fatal CHD [56-59], and the pooled analysis that reported a $39 \%$ reduction for fatal CHD [61].

Summary Statement for Epidemiologic Studies of Nut Consumption, CHD Risk and Cardiovascular Mortality

Strong and consistent evidence from four large US epidemiologic studies conducted over the past two decades has shown an association between the increased frequency of weekly nut intake and reduced CHD risk. The pooled data from these studies supports the recommendation for frequent nut intake to reduce CHD risk among the US population. The PREDIMED trial, which featured a large prospective cohort outside of the US, also found similar beneficial outcomes between the frequency of nut intake and diminished CHD risk and cardiovascular mortality.

\section{Nuts and Primary and Secondary Prevention of CVD}

The findings reported by the PREDIMED team have provided support for the use of nuts in the primary prevention of CVD among high-risk individuals [63••]. The PREDIMED trial's primary endpoint was the combination of the rate of several major cardiovascular events, i.e., myocardial infarction, stroke or cardiovascular death. Compared to the low-fat control group, a multivariable adjusted hazard ratio (HR) of $0.72(95 \% \mathrm{CI}$, 0.54-0.96) was observed for the group assigned to consumption of a Mediterranean diet with mixed nuts, and an HR of 0.70 (95\% CI 0.54-0.92) was found for the group assigned to consumption of a Mediterranean diet with extra-virgin olive oil. The results of this landmark trial have significant public health implications in light of the lack of energy restrictions 
during the trial and the challenges of individuals struggling with weight management for the primary prevention of CVD.

The findings of the PREDIMED trial complement those of the Lyon Diet Heart Study, which was a secondary CHD prevention trial that suggested that an unrestricted-energy Mediterranean diet rich in ALA (found in high levels in walnuts) could reduce the risk of recurrence [64]. Specifically, supplementation of the Mediterranean diet (rich in oleic acid/ MUFA, low in SFA, and low in n-6 fatty acids) with $1 \mathrm{~g}$ per day of very long-chain n-3 fatty acids was associated with a 50-70\% reduced risk of recurrence after 4 years of follow-up among participants with CHD. Similar findings were noted in the Cholesterol and Recurrent Events (CARE) Study, which evaluated the cardio-protective effect of frequent nut consumption in a population that had previously experienced a non-fatal myocardial infarction [65]. These investigators found that those consuming greater than one serving of nuts per week experienced a $25 \%$ reduced risk of total CHD compared to those who rarely consumed nuts or consumed less than one serving per month.

\section{Summary Statement for Nuts and Primary and Secondary Prevention of CVD}

A recent large trial conducted in Spain (PREDIMED) was very successful in using an unrestricted-energy Mediterranean-type background diet with mixed nuts for the primary prevention of CVD. Further, use of an ALA supplement or frequent nut consumption has been shown to prevent recurrent CHD events. Hence, it is of paramount importance to design public health messages regarding the appropriate use of nut-based dietary patterns or fatty acid dietary strategies for the primary and secondary prevention of CVD. In light of an ALA supplement having been found to be effective in a secondary prevention trial, a future trial to evaluate the effects of a walnut-enriched, unrestricted-energy Mediterranean diet would be of interest to evaluate whether daily walnut intake could achieve similar or heightened findings.

\section{Conclusions}

Significant evidence has accumulated demonstrating the cardioprotective benefits from frequent nut consumption, beginning with the findings from the 1992 Loma Linda University AHS-I to the 2013 release of the PREDIMED trial results. Nuts are nutrient-dense whole plant foods that have the potential for the primary and secondary prevention of CVD and reducing cardiovascular mortality. Government funded trials are warranted to determine the synergistic potential of nuts on CVD biomarkers and outcomes using a variety of background dietary patterns and combinations of nuts. Public health messages recommending frequent nut intake should be framed in the context of the findings from amply powered trials, and should consider the populations evaluated and their respective background dietary patterns.

\section{Compliance with Ethics Guidelines}

Conflict of Interest Joan Sabaté has received previous research grant support from the California Walnut Commission and the National Peanut Board.

Michelle Wien has received previous research grant support from the Almond Board of California.

Human and Animal Rights and Informed Consent This article does not contain any studies with human or animal subjects performed by any of the authors.

\section{References}

Papers of particular interest, published within the past 3 years, have been highlighted as:

- Of importance

•• Of major importance

1. - World Health Organization. Death and disability due to CVDs (heart attacks and strokes). In Global Atlas on cardiovascular disease prevention and control. In: Mendis S, Puska P, Norrving editors. Geneva: WHO Press; 2011:8-13. This book chapter presents current global statistics for death and disability due to cardiovascular diseases.

2. Kris-Etherton PM, Yu-Poth S, Sabate J, et al. Nuts and their bioactive constituents: effects on serum lipids and other factors that affect disease risk. Am J Clin Nutr. 1999;70:504S-11S.

3. Calder PC, Yaqoob P. Omega-3 polyunsaturated fatty acids and human health outcomes. Biofactors. 2009;35:266-72.

4. Kaminski WE, Jendraschak E, Kiefl R, et al. Dietary omega-3 fatty acids lower levels of platelet-derived growth factor mRNA in human mononuclear cells. Blood. 1993;81:1871-9.

5. Brufau G, Boatella J, Rafecas M. Nuts: source of energy and macronutrients. Br J Nutr. 2006;96 Suppl 2:S24-8.

6. Huynh NN, Chin-Dusting J. Amino acids, arginase and nitric oxide in vascular health. Clin Exp Pharmacol Physiol. 2006;33:1-8.

7. Anderson JW, Johnstone BM, Cook-Newell ME. Meta-analysis of the effects of soy protein intake on serum lipids. N Engl J Med. 1995;333:276-82.

8. Jenkins DJ, Wolever TM, Spiller G, Buckley G, Lam Y, Jenkins AL, et al. Hypocholesterolemic effect of vegetable protein in a hypocaloric diet. Atherosclerosis. 1989;78:99-107.

9. Salas-Salvado J, Bullo M, Perez-Heras A, Ros E. Dietary fibre, nuts and cardiovascular diseases. Br J Nutr. 2006;96 Suppl 2:S46-51.

10. Blomhoff R, Carlsen MH, Andersen LF, et al. Health benefits of nuts: potential role of antioxidants. Br J Nutr. 2006;96 Suppl 2:S52-60.

11. Ostlund Jr RE. Phytosterols in human nutrition. Annu Rev Nutr. 2002;22:533-49.

12. Appel LJ, Moore TJ, Obarzanek E, et al. A clinical trial of the effects of dietary patterns on blood pressure. DASH collaborative research group. N Engl J Med. 1997;336:1117-24.

13. Fraser GE. Nut consumption, lipids, and risk of a coronary event. Clin Cardiol. 1999;22:11-5. 
14. Champagne CM. Magnesium in hypertension, cardiovascular disease, metabolic syndrome, and other conditions: a review. Nutr Clin Pract. 2008;23:142-51.

15. Houtman JP. Trace elements and cardiovascular diseases. J Cardiovasc Risk. 1996;3:18-25.

16. Sabaté J, Fraser GE, Burke K, et al. Effects of walnuts on serum lipid levels and blood pressure in normal men. N Engl J Med. 1993;328:603-7.

17. U.S. Food and Drug Administration: Qualified health claims: Letter of enforcement discretion-nuts and coronary heart disease (DocketNo.02P-0505). Available at http://www.fda.gov/Food/ LabelingNutrition/LabelClaims/QualifiedHealthClaims/ucm072926. htm. Accessed August 2013.

18. •• Sabaté J, Oda K, Ros E: Nut consumption and blood lipid levels: a pooled analysis of 25 intervention trials. Arch Intern Med 2010, 170: 821-827. This article presents important pooled analysis data from 25 nut intervention trials conducted in seven countries showing that nut consumption lowers cornonary heart disease risk.

19. Banel DK, Hu FB. Effects of walnut consumption on blood lipids and other cardiovascular risk factors: a meta-analysis and systematic review. Am J Clin Nutr. 2009;90:56-63.

20. Daniels SR, Arnett DK, Eckel RH, et al. Overweight in children and adolescents: pathophysiology, consequences, prevention, and treatment. Circulation. 2005;111:1999-2012.

21. • Maranhao PA, Kraemer-Aguiar LG, de Oliveira CL,et al.: Brazil nuts intake improves lipid profile, oxidative stress and microvascular function in obese adolescents: a randomized controlled trial. Nutr Metab 2011, 8:32. This article presents some of the first data to document the beneficial effects of Brazil nut consumption among obese adolescents.

22. Coates AM, Howe PR. Edible nuts and metabolic health. Curr Opin Lipidol. 2007;18:25-30.

23. Lopez-Uriarte P, Bullo M, Casas-Agustench P, et al. Nuts and oxidation: a systematic review. Nutr Rev. 2009;67:497-508.

24. Nash SD, Nash DT. Nuts as part of a healthy cardiovascular diet. Curr Atheroscler Rep. 2008;10:529-35.

25. Ros E. Nuts and novel biomarkers of cardiovascular disease. Am J Clin Nutr. 2009;89:1649S-56S.

26. Reaven PD, Witztum JL. Oxidized low density lipoproteins in atherogenesis: role of dietary modification. Annu Rev Nutr. 1996;16:51-71.

27. Berry SE, Tydeman EA, Lewis HB, et al. Manipulation of lipid bioaccessibility of almond seeds influences postprandial lipemia in healthy human subjects. Am J Clin Nutr. 2008;88:922-9.

28. Cortes B, Nunez I, Cofan M, et al. Acute effects of high-fat meals enriched with walnuts or olive oil on postprandial endothelial function. J Am Coll Cardiol. 2006;48:1666-71.

29. Jenkins DJ, Kendall CW, Josse AR, et al. Almonds decrease postprandial glycemia, insulinemia, and oxidative damage in healthy individuals. J Nutr. 2006;136:2987-92.

30. Torabian S, Haddad E, Rajaram S, et al. Acute effect of nut consumption on plasma total polyphenols, antioxidant capacity and lipid peroxidation. J Hum Nutr Diet. 2009;22:64-71.

31. - Hudthagosol C, Haddad EH, McCarthy K, et al.: Pecans acutely increase plasma postprandial antioxidant capacity and catechins and decrease LDL oxidation in humans. J Nutr 2011, 141:56-62. This article features interesting data showing that bioactive constituents of pecans are absorbable and contribute to postprandial antioxident defenses.

32. • Kay CD, Gebauer SK, West SG, et al.: Pistachios increase serum antioxidants and lower serum oxidized-LDL in hypercholesterolemic adults. J Nutr 2010, 140:1093-1098. This article presents data showing that consumption of a pistachio-enriched healthy diet confers cardioprotective benefits beyond lipid-lowering, i.e., decreased oxidized-LDL.
33. Fito M, Guxens M, Corella D, et al. Effect of a traditional Mediterranean diet on lipoprotein oxidation: a randomized controlled trial. Arch Intern Med. 2007;167:1195-203.

34. • Lopez-Uriarte P, Nogues R, Saez G, et al.: Effect of nut consumption on oxidative stress and the endothelial function in metabolic syndrome. Clin Nutr 2010, 29:373-380. This article presents some of the first data showing that despite the possible adverse pro-oxidant effect of consuming PUFA-rich nuts, no deleterious effect on biomarkers of oxidative stress or endothelial function in patients with metabolic syndrome was observed.

35. Estruch R, Martinez-Gonzalez MA, Corella D, et al. Effects of a Mediterranean-style diet on cardiovascular risk factors: a randomized trial. Ann Intern Med. 2006;145:1-11.

36. Ros E, Nunez I, Perez-Heras A, et al. A walnut diet improves endothelial function in hypercholesterolemic subjects: a randomized crossover trial. Circulation. 2004;109:1609-14.

37. - Rajaram S, Connell KM, Sabaté J: Effect of almond-enriched high-monounsaturated fat diet on selected markers of inflammation: a randomised, controlled, crossover study. Br J Nutr 2010, 103:907-912. This article provides data on the effects of almond consumption on markers of inflammation, showing reductions in E-selectin and C-reactive protein in the context of null results for IL-6 and fibrinogen.

38. Jimenez-Gomez Y, Lopez-Miranda J, Blanco-Colio LM, et al. Olive oil and walnut breakfasts reduce the postprandial inflammatory response in mononuclear cells compared with a butter breakfast in healthy men. Atherosclerosis. 2009;204:e70-6.

39. Mena MP, Sacanella E, Vazquez-Agell M, et al. Inhibition of circulating immune cell activation: a molecular antiinflammatory effect of the Mediterranean diet. Am J Clin Nutr. 2009;89:248-56.

40. Kay CD, Kris-Etherton PM, West SG. Effects of antioxidant-rich foods on vascular reactivity: review of the clinical evidence. Curr Atheroscler Rep. 2006;8:510-22.

41. Vita JA. Polyphenols and cardiovascular disease: effects on endothelial and platelet function. Am J Clin Nutr. 2005;81:292S-7S.

42. Gornik HL, Creager MA. Arginine and endothelial and vascular health. J Nutr. 2004;134:2880S-7S. discussion 2895S.

43. de Koning EJ, Rabelink TJ. Endothelial function in the post-prandial state. Atheroscler. 2002;3:S11-6.

44. Brown AA, Hu FB. Dietary modulation of endothelial function: implications for cardiovascular disease. Am J Clin Nutr. 2001;73:673-86.

45. Sanderson P, Sattar N, Olthof M, et al. Dietary lipids and vascular function: UK Food Standards Agency workshop report. Br J Nutr. 2004;91:491-500.

46. West SG. Effect of diet on vascular reactivity: an emerging marker for vascular risk. Curr Atheroscler Rep. 2001;3:446-55.

47. Deanfield JE, Halcox JP, Rabelink TJ. Endothelial function and dysfunction: testing and clinical relevance. Circulation. 2007;115: 1285-95.

48. • Ma Y, Njike VY, Millet J, et al.: Effects of walnut consumption on endothelial function in type 2 diabetic subjects: a randomized controlled crossover trial. Diabetes Care 2010, 33:227-232. This report was the first to demonstrate that a walnut-enriched ad libitum diet improves endothelial function in individuals with type 2 diabetes, which suggests that a simple dietary nut intervention may confer a reduction in overall cardiovascular risk.

49. •• Official Journal of the European Union. Available at http://eur-lex. europa.eu/LexUriServ/LexUriServ.do?uri=OJ:L:2012: 136:FULL:EN:PDF. Accessed August 2013. This journal contains a notice for a specific health claim for walnuts in the European Union that states "Walnuts contribute to the improvement of the elasticity of the blood vessels," and may be used only for food that provides a daily intake of 30 grams of walnuts. 
50. Villegas R, Gao YT, Yang G, et al. Legume and soy food intake and the incidence of type 2 diabetes in the Shanghai Women's Health Study. Am J Clin Nutr. 2008;87:162-7.

51. - Kochar J, Gaziano JM, Djousse L: Nut consumption and risk of type II diabetes in the Physicians' Health Study. Eur J Clin Nutr 2010, 64:75-79. This report shows data that does not support an association between nut consumption and incident type 2 diabetes among a prospective cohort of 20,224 male participants enrolled in the Physicians' Health Study.

52. Barcelo F, Perona JS, Prades J, et al. Mediterranean-style diet effect on the structural properties of the erythrocyte cell membrane of hypertensive patients: the Prevencion con Dieta Mediterranea Study. Hypertension. 2009;54:1143-50.

53. Fuster V, Ryden LE, Cannom DS, ACC/AHA/ESC, et al. Guidelines for the Management of Patients with Atrial Fibrillation: a report of the American College of Cardiology/American Heart Association Task Force on Practice Guidelines and the European Society of Cardiology Committee for Practice Guidelines (Writing Committee to Revise the 2001 Guidelines for the Management of Patients With Atrial Fibrillation): developed in collaboration with the European Heart Rhythm Association and the Heart Rhythm Society. Circulation. 2006;2006(114):e257-354.

54. Go AS, Hylek EM, Phillips KA, et al. Prevalence of diagnosed atrial fibrillation in adults: national implications for rhythm management and stroke prevention: the AnTicoagulation and Risk Factors in Atrial Fibrillation (ATRIA) Study. JAMA. 2001;285:2370-5.

55. - Khawaja O, Gaziano JM, Djousse L: Nut consumption and risk of atrial fibrillation in the Physicians' Health Study. Nutr J 2012, 11:17. This report shows data that does not support an association between nut consumption and incident atrial fibrillation among a prospective cohort of 21,054 male participants enrolled in the Physicians' Health Study.

56. Fraser GE, Sabaté J, Beeson WL, et al. A possible protective effect of nut consumption on risk of coronary heart disease. The Adventist Health Study Arch Intern Med. 1992;152:1416-24.
57. Hu FB, Stampfer MJ, Manson JE, et al. Frequent nut consumption and risk of coronary heart disease in women: prospective cohort study. BMJ. 1998;317:1341-5.

58. Albert CM, Gaziano JM, Willett WC, et al. Nut consumption and decreased risk of sudden cardiac death in the Physicians' Health Study. Arch Intern Med. 2002;162:1382-7.

59. Ellsworth JL, Kushi LH, Folsom AR. Frequent nut intake and risk of death from coronary heart disease and all causes in postmenopausal women: the Iowa Women's Health Study. Nutr Metab Cardiovasc Dis. 2001;11:372-7.

60. Kelly Jr JH, Sabaté J. Nuts and coronary heart disease: an epidemiological perspective. Br J Nutr. 2006;96 Suppl 2:S61-7.

61. Kris-Etherton PM, Hu FB, Ros E, et al. The role of tree nuts and peanuts in the prevention of coronary heart disease: multiple potential mechanisms. J Nutr. 2008;138:1746S-51S.

62. •- Guasch-Ferre M, Bullo M, Martinez-Gonzalez MA, et al.: Frequency of nut consumption and mortality risk in the PREDIMED nutrition intervention trial. BMC Med 2013, 11:164. This article presents findings from 7,216 participants enrolled in the PREDIMED trial that showed that participants that consumed $>3$ weekly servings of total nuts, walnuts or other nuts (excluding walnuts) at baseline had significant reductions in cardiovascular mortality of 55, 47 and $58 \%$, respectively.

63. •- Estruch R, Ros E, Salas-Salvado J, et al.: Primary prevention of cardiovascular disease with a Mediterranean diet. N Engl J Med 2013, 368:1279-1290. This article presents findings from 7,447 participants enrolled in the PREDIMED trial that demonstrated that a Mediterranean diet supplemented with mixed nuts daily lowers the risk of CVD by $30 \%$.

64. de Lorgeril M, Salen P, Martin JL, et al. Mediterranean diet, traditional risk factors, and the rate of cardiovascular complications after myocardial infarction: final report of the Lyon Diet Heart Study. Circulation. 1999;99:779-85.

65. Brown L, Rosber B, Willet W, et al. Nut consumption and risk of recurrent coronary heart disease. FASEB J. 1999;13:A538. 